\title{
ON THE DISTORTION AND CORRESPONDENCE UNDER QUASICONFORMAL MAPPINGS IN SPACE
}

\author{
KAZUO IKOMA
}

\section{Introduction}

Recently many important results on rings and quasiconformal mappings in space have been obtained by B. V. Šabat [9], F. W. Gehring [3], J. Väisälä [11] and others. The modulus of a ring in space can be defined in three apparently different but essentially equivalent ways. (See Gehring [4]). In the theory of quasiconformal mappings in space, some properties for moduli of rings in space play an important role, because the method by means of moduli acts also as a substitute in space for the Riemann mapping theorem which does not hold in space.

In this paper, we shall give the extremal condition for spherical rings, an extension of Grötzsch-Šabat's theorem and an example of space $K$-quasiconformal mappings constructed by associating with certain plane $K$-quasiconformal ones. By using these results, the monotoneity for moduli of space rings (B. Fuglede [1]) and the extremality and estimates for moduli of the space Grötzsch and Teichmüller rings (Gehring [2]) as our main tools, we shall establish mainly the space forms corresponding to our previous results ([5], [6]) of plane $K$ quasiconformal mappings. That is to say, we are concerned with the Schwarz's lemma for space $K$-quasiconformal mappings, some distortions in these mappings (under a certain normalization at the origin) and a criterion for two sets corresponding to each other by such mappings to be of the same dimensional Hausdorff measure zero, where it is remarked that even if one of such mappings transforms a plane domain into another plane domain, its correspondence between these plane domains does not always induce a plane $K$-quasiconformal mapping.

For the sake of convenience, we shall mostly restrict rings and $K$-quasiconformal mappings to the 3-dimensional space.

The author wishes to express his hearty gratitude to Professors K. Noshiro

Received June 1, 1964. 
and T. Kuroda for their kind encouragement and guidance during his researches.

\section{Preliminaries}

1. A space ring $R^{3}$ is defined as a domain whose complement in the Moebius space, that is, the finite Euclidean 3 -space plus the point at infinity consists of two components $C_{0}$ and $C_{1}$ such that $C_{0}$ is bounded and $C_{1}$ is bounded or unbounded. Then the boundaries $B_{0}$ and $B_{1}$ of $C_{0}$ and $C_{1}$ are the boundary components of $R^{3}$.

For each non-negative Borel measurable function $\rho(x)$ defined in a space ring $R^{3}$, if we put

$$
L(\rho)=\inf _{\gamma} \int_{\gamma} \rho d s, \quad V(\rho)=\iiint_{R^{3}} \rho^{3} d \tau,
$$

where $\gamma$ denotes any locally rectifiable curve which joins the boundary components $B_{0}, B_{1}$ in $R^{3}$, then the quantity

$$
\left\{4 \pi \sup _{\rho} \frac{L(\rho)^{3}}{V(\rho)}\right\}^{-\frac{1}{2}}
$$

is called the modulus of $R^{3}$, which is denoted by $\bmod R^{3}$.

It is seen by Fuglede [1] that

$$
\bmod R_{1}^{3} \leqq \bmod R_{2}^{3} \quad \text { (monotoneity property) }
$$

if the ring $R_{1}^{3}$ separates the boundary components of the ring $R_{2}^{3}$.

2. For each $a>1$, let $R_{G}^{3}(a)$ be the space ring whose complementary components consist of the sphere $|x| \leqq 1$ and the ray $a \leqq x_{1} \leqq \infty, x_{2}=x_{3}=0$, which is called the space Grötzsch ring. Similarly for each $b>0$, let $R_{T}^{3}(b)$ be the space ring bounded by the segment $-1 \leqq x_{1} \leqq 0, x_{2}=x_{3}=0$ and the ray $b \leqq x_{1}$ $\leqq \infty, x_{2}=x_{3}=0$, which is called the space Teichmüller ring. If we set

$$
\bmod R_{G}^{3}(a)=\log \mathscr{D}_{3}(a), \quad \bmod R_{T}^{3}(a)=\log \Psi_{3}(a),
$$

then clearly $\Phi_{3}(a)$ and $\Psi_{3}(a)$ are non-decreasing in $a$, and it has been proved by Gehring [2] that it holds

$$
a \leqq \Phi_{3}(a) \leqq \lambda a
$$

for $1<a<\infty$, where $\lambda$ is such a constant as $4 \leqq \lambda \leqq 12.4 \ldots \ldots$

As in the plane case, the rings $R_{G}^{3}$ and $R_{T}^{3}$ have the following extremal 
properties respectively.

Lemma 1. (Šabat [9]). Suppose that $R$ is a space ring whose $C_{0}$ contains the sphere $\left|x-x_{0}\right| \leqq a$ and whose $C_{1}$ is unbounded and contains at least one point at distance $b(>a)$ from $x_{0}$, then there holds

$$
\bmod R \leqq \log \Phi_{3}\left(\begin{array}{l}
b \\
a
\end{array}\right)
$$

where the equality holds when $C_{0}$ is the sphere $\left|x-x_{0}\right| \leqq a$ and $C_{1}$ is the radial ray from one point on $\left|x-x_{0}\right|=b$ to the point at infinity.

Lemma 2. (Gehring [2]). Suppose that $R$ is a space ring whose $C_{0}$ contains $a$ point $x_{1}$ and at least one point at distance a from $x_{0}$ and whose $C_{1}$ is unbounded and contains at least one point at distance $b$ from $x_{0}$, then there holds

$$
\bmod R \leqq \log \Psi_{3}\left(\begin{array}{c}
b \\
a
\end{array}\right)
$$

where the equality holds when $C_{0}$ is a segment $x_{0} x_{a}$ with length $a$ and $C_{1}$ is the ray starting from one point at distance $b$ from $x_{0}$ and reaching the point at infinity on the oriented half line $\overrightarrow{x_{a} x_{0}}$.

3. Now, a $K$-quasiconformal mapping in space is defined in terms of moduli of rings in space as follows.

Geometric definition. A homeomorphism $y(x)$ of a domain $D$ is called a $K$-quasiconformal mapping, $1 \leqq K<\infty$, if the modulus condition

$$
\frac{1}{K} \bmod R \leqq \bmod R^{\prime} \leqq K \bmod R
$$

is satisfied for all bounded rings $R$ with $\bar{R} \subset D$, where $R^{\prime}$ and $\bar{R}$ denote the image and the closure of $R$ respectively.

$K$-quasiconformality equivalent to this is analytically stated as follows.

Analytic Definition. A homeomorphism $y(x)$ of a domain $D$ is said to be a $K$-quasiconformal mapping, $1 \leqq K<\infty$, provided that it is absolutely continuous on almost all line segments, parallel to each of the coordinate axes, in each sphere $U$ with $\bar{U} \subset D$, and holds

$$
\frac{1}{K^{2}} \max \left|y^{\prime}(x)\right|^{3} \leqq|J(x)| \leqq K^{2} \min \left|y^{\prime}(x)\right|^{3}
$$


almost everywhere in $D$, where $y^{\prime}(x)$ and $J(x)$ denote the directional derivative and Jacobian of $y(x)$ respectively.

It is well known that the restriction of a Moebius transformation to a space domain is equivalent to a 1 -quasiconformal mapping of its domain.

Remark. Since Väisälä [11] defined modulus of a space ring by the quantity $4 \pi \sup _{\rho}\left\{L(\rho)^{3} / V(\rho)\right\}$, a mapping is $K$-quasiconformal in his sense if and only if it is $K^{\frac{1}{2}}$-quasiconformal by the above definitions.

A homeomorphism $y(x)$ of a domain is referred to a $K$-quasiconformal mapping in the classical sense, if it is continuously differentiable with $J(x)>0$ and satisfies

$$
\max \left(\frac{\max \left|y^{\prime}(x)\right|^{3}}{|J(x)|}, \frac{|J(x)|}{\min \left|y^{\prime}(x)\right|^{3}}\right) \leqq K^{2}
$$

everywhere in $D$ (except possibly for a set being isolated and relatively closed in $D$ ). It is found by arguments in Väisälä [11] that a $K$-quasiconformal mapping in the classical sense is also $K$-quasiconformal according to the preceding two definitions.

Similarly, a ring $R^{n}$ in the Moebius $n$-dimensional space is defined, and its modulus is defined in terms of $L(\rho), V(\rho)$ corresponding to $R^{n}$ by

$$
\left\{\frac{2 \pi^{\frac{n}{2}}}{\Gamma\left(\begin{array}{c}
n \\
2
\end{array}\right)} \sup _{\rho} \frac{L(\rho)^{n}}{V(\rho)}\right\}^{\frac{1}{n-1}}
$$

Further, in terms of these rings, a $K$-quasiconformal mapping of a domain in the $n$-dimensional space is also defined. The monotoneity property of the modulus of a ring in higher dimensional space remains also valid. (Fuglede [1]).

4. Gehring ([2], Theorem 3) showed the analogue of a theorem of G. M. Golusin which gives an upper bound for the modulus of a ring. But the necessary condition for the ring that its modulus attains the upper bound is not clear.

For the moduli of the Grötzsch and Teichmüller rings, Gehring [4] showed that

$$
\Phi_{2}(a) \leqq \emptyset_{3}(a) \text { and } \Psi_{2}(a) \leqq \Psi_{3}(a)
$$


where $\log \Phi_{2}(a)$ and $\log \Psi_{2}(a)$ denote the moduli of the Grötzsch and Teichmüller rings in the plane, respectively.

Here, we deal with the following special case where the necessary condition for the space ring to be extremal in the above sense and the relation between moduli of the plane ring with the symmetric axis and of the space ring obtained by revolving it about its axis can be simultaneously clarified. The plane ring $R^{2}$ bounded by two circles one of which lies in the other is called a circular ring, and the space ring $R^{3}$ obtained by revolving $R^{2}$ around its central line, i.e. symmetric axis, is referred to the spherical ring corresponding to the circular ring $R^{2}$. In particular, it is well known that for a concentric circular ring $R_{0}^{2}$ : $a<|z|<b$ and a concentric spherical ring $R_{0}^{3}: a<|x|<b$, it holds

$$
\bmod R_{0}^{2}=\log \frac{b}{a}=\bmod R_{0}^{3} .
$$

We have the following

Lemмa 3. The modulis of the spherical ring $R$ bounded by two spherical surfaces with radii $r, b(r<b)$ is not greater than $\log \frac{b}{r}$. Further, mod $R$ attains the maximum value $\log \frac{b}{r}$ if and only if $R$ is the concentric spherical ring $R_{0}: r<|x|<b$.

Proof. Without loss of generality, we may assume that $R$ is a spherical ring bounded by $x_{1}^{2}+x_{2}^{2}+x_{3}^{2}=1$ and $\left(x_{1}-c\right)^{2}+x_{2}^{2}+x_{3}^{2}=r^{2}(0 \leqq c<1-r)$. Then, by some elementary computations, it is seen that $R$ can be mapped onto a concentric spherical ring $r^{\prime}<\sqrt{y_{1}^{2}+y_{2}^{2}+y_{3}^{2}}<1$ by the restriction of a composite mapping of

$$
\begin{gathered}
x_{1}-\frac{r^{\prime}(c-r)+1}{c+r^{\prime}-r}=X_{1}, \quad x_{2}=X_{2}, \quad x_{3}=X_{3}, \\
y_{j}=\frac{\left(\frac{r^{\prime}(c-r)+1}{c+r^{\prime}-r}\right)^{2}-1}{X_{1}^{2}+X_{2}^{2}+X_{3}^{2}} X_{j} \quad(j=1,2,3), \\
-\left(y_{1}+\frac{r^{\prime}(c-r)+1}{c+r^{\prime}-r}\right)=Y_{1}, \quad y_{2}=Y_{2}, y_{3}=Y_{5},
\end{gathered}
$$

where $r^{\prime}=\frac{1}{2 r}\left\{r^{2}+1-c^{2}-\sqrt{\left(r^{2}+1-c^{2}\right)^{2}-4 r^{2}}\right\}$. Since this composite mapping is a Moebius transformation and so a 1-quasiconformal mapping, it holds that $\bmod R=\log \frac{1}{r^{\prime}}$. On the other hand, it is clear that $\bmod R_{0}=\log \frac{1}{r}$. Moreover, 
it can be easily verified that the inequality $\frac{1}{r^{\prime}} \leqq \frac{1}{r}$ holds and the equality holds if and only if $c=0$.

From the above proof of Lemma 3, we can find immediately the following

LEMma 4. The modulus of the spherical ring corresponding to a circular ring is equal to that of the circular ring.

\section{Distortion under quasiconformal mappings}

5. The so-called lemma of Schwarz on bounded and regular functions in a circular disc gives two precise estimates, at each point in the circular disc, the first of which is made for absolute values of these functions and the second of which is made for absolute values of their derivatives. A space analogue corresponding to the first estimate in the lemma of Schwarz was shown by Šabat, who states only $K$-quasiconformal mappings of $|x|<1$ "onto" itself. We enunciate here, for the later use, in a slightly more general form replaced "onto" by "into", and prove it to make sure.

THEOREM 1. Let $y=y(x)$ be any K-quasiconformal mapping of $|x|=\sqrt{x_{1}^{2}+x_{2}^{2}+x_{3}^{2}}$ $<1$ such that $|y(x)|<1$ and $y(0)=0$. Then for any $0<|x|<1$,

$$
\left\{\Phi_{3}\left(\frac{1}{|x|}\right)\right\}^{\stackrel{1}{K}} \leqq \Phi_{3}\left(\begin{array}{c}
1 \\
|y|
\end{array}\right) .
$$

Proof. Let $R_{x}$ denote the unit sphere $|x|<1$ slit along the segment from the origin to the point $x$, so that $\bmod R_{x}=\log \Phi_{3}\left(\frac{1}{|x|}\right)$. Similarly, define the ring $R_{y}$ in the unit sphere $|y|<1: \bmod R_{y}=\log \Phi_{3}\left(\frac{1}{|y|}\right)$. Since the image $y\left(R_{x}\right)$ of $R_{x}$ by $y=y(x)$ is a domain in $|y|<1$ slit along a curve from the origin to the point $y(x)$, we have by Lemma 1 and by the modulus condition,

$$
\frac{1}{K} \bmod R_{x} \leqq \bmod y\left(R_{x}\right) \leqq \bmod R_{y},
$$

whence follows our-assertion.

Applying Theorem 1 to the inverse of a mapping in Theorem 1, we have

CoROLlaRY 1. Let $y=y(x)$ be any K-quasiconformal mapping of a domain in $|x|<1$ onto $|y|<1$ with $y(0)=0$. Then for any $0<|x|<1$, 


$$
\Phi_{3}\left(\frac{1}{|y|}\right) \leqq\left\{\Phi_{3}\left(\frac{1}{|x|}\right)\right\}^{K}
$$

In particular, we have

Corollary 2. (Šabat [9]). Let $y=y(x)$ be any $K$-quasiconformal mapping of $|x|<1$ onto $|y|<1$ with $y(0)=0$. Then for any $0<|x|<1$,

$$
\left\{\Phi_{3}\left(\frac{1}{|x|}\right)\right\}^{-\frac{1}{K}} \leqq \Phi_{3}\left(\frac{1}{|y|}\right) \leqq\left\{\Phi_{3}\left(\frac{1}{|x|}\right)\right\}^{K} .
$$

Remark. An extremal $K$-quasiconformal mapping which induces the equality for each $x$ in $0<|x|<1$ in each of the above estimates has not yet been found.

6. Next, we consider a space form corresponding to the second estimate in the lemma of Schwarz.

THEOREM 2. Let $y=y(x)$ be any K-quasiconformal mapping of $|x|<1$ such that $|y|<1, y(0)=0$ and such that $y(x)$ maps each radius of $|x|<1$ onto a curve which is normal to the image of each surface $|x|=r(<1)$. Then

$$
\liminf _{x \rightarrow 0} \frac{|y(x)|}{|x|^{\frac{1}{K}}} \leqq 1,
$$

where the equality holds only if $y=y\left(x\left(r, \theta_{1}, \theta_{2}\right)\right): y_{1}=r^{\frac{1}{K}} \cos \theta_{1}, y_{2}=r^{\frac{1}{A}} \sin \theta_{1}$ $\cos \left(\theta_{2}+c\right), y_{3}=r^{\kappa} \sin \theta_{1} \sin \left(\theta_{2}+c\right)$ with a real constant $c$.

Proof. Denote by $\sum_{r}$ the image of the spherical surface $|x|=r$ under $y=y(x)$, and by $A(r)$ and $V(r)$ the surface area of $\Sigma_{r}$ and the volume bounded by $\sum_{r}$ respectively. Let $N(x)$ be the directional derivative in the radial direction: $N(x)=\lim _{h \rightarrow 0}\{y(x+h x)-y(x)\} / h x$, where $h$ is a real number, and let $J(x)$ be Jacobian of $y(x)$. Then by some geometric considerations, it is found that $|J(x)| /|N(x)|$ is the ratio between corresponding elements of area on $\sum$ - and $|x|=r$. Hence we have for almost every $r(0<r<1)$,

$$
A(r)=\iint_{\Sigma_{r}} d \sigma=\iint_{\{x\}=r}\left|\frac{J(x)}{N(x)}\right| d \omega .
$$

Applying Hölder's inequality, we have

$$
A(r)^{2} \leqq\left(\iint_{|x|=r} d \omega\right)^{2} \iint_{|x|=r}\left|\frac{J(x)}{N(x)}\right|^{3} d \omega .
$$


In view of the analytic definition of a $K$-quasiconformal mapping in $\S 3$, we see $\left\{|J(x)| /|N(x)|^{3}\right\}^{\frac{1}{2}} \leqq K$ almost everywhere. Hence we have

$$
A(r)^{2} \leqq 2 \sqrt{\pi} r K \iint_{|x|=r}|J(x)| d \omega=2 \sqrt{\pi} r K \frac{d V(r)}{d r} .
$$

Taking account of the well-known isoperimetric inequality

$$
A(r)^{3}-36 \pi V(r)^{2} \geqq 0
$$

(vid., e.g. [8]), we obtain for almost all $0<r<1$,

$$
\frac{d V(r)}{d r} \geqq \frac{3 V(r)}{r K} .
$$

and hence

$$
\frac{d}{d r}\left\{\frac{V(r)}{r^{\frac{3}{3}}}\right\} \geqq 0
$$

Therefore $V(r) / r^{\frac{1}{K}}$ is a non-decreasing function of $r$. Thus we get

$$
V(\boldsymbol{r}) / \boldsymbol{r}^{3} \boldsymbol{x}^{-} \leqq V(1)=\frac{4}{3} \pi
$$

Put $\min _{|x|=r}|y(x)|=m(r)$. Then it is evident from $y(0)=0$ that $\frac{4}{3} \pi(m(r))^{3} \leqq$ $V(r)$. Thus we obtain

$$
\begin{aligned}
& \liminf _{x \rightarrow 0} \frac{|y(x)|}{|x|^{\frac{1}{K}}}=\liminf _{r \rightarrow 0} \frac{m(r)}{r^{\frac{1}{K}}} \\
\leqq & \liminf _{r \rightarrow 0}\left\{3 V(r) / 4 \pi r^{\frac{3}{K}}\right\}^{\frac{1}{3}} \leqq 1 .
\end{aligned}
$$

In the next place, we consider the extremal case where $\lim _{x \rightarrow 0} \inf |y(x)| /|x|^{-1}$ $=1$. Then it is noted that the equality holds in (3), because $V(r) / r^{{ }^{K}}$ is a non-decreasing function of $r$. Hence the signs of equality hold in (2) and (1) also. This implies that the image of $|x|=r(\leqq 1)$ by $y=y(x)$ is a spherical surface with radius $r^{\stackrel{1}{K}}$.

Now, the modulus of the spherical ring bounded by $\Sigma_{r}$ and $|y|=1$ is not less than $\frac{1}{K} \log \frac{1}{r}$ in view of the modulus condition, while its modulus is not greater than $\frac{1}{K} \log \frac{1}{r}$ as is seen by making use of Lemma 3, from which follows that its modulus is equal to $\frac{1}{K} \log \frac{1}{r}$. We can see by Lemma 3 again that the center of $\sum_{r}$ for $0<r<1$ is always $y=0$. Hence, an extremal mapping 
$y=y(x): y_{1}=\rho \cos \phi_{1}, \quad y_{2}=\rho \sin \phi_{1} \cos \phi_{2}, y_{3}=\rho \sin \phi_{1} \sin \phi_{2}$ maps the surface $|x|=r$ for each $0 \leqq r \leqq 1$ onto the surface $|y|=r^{\frac{1}{K}}$. Thus we have $\rho=r^{K}$.

Since the equality holds also in Hölder's inequality (1), we have

$$
|J(x)| /|N(x)|=\sin \phi_{1} d \phi_{1} d \phi_{2} / \sin \theta_{1} d \theta_{1} d \theta_{2}=\text { constant }
$$

for each fixed $r$ and almost every $\theta_{1}, \theta_{2}$ such that $0<r<1,0 \leqq \theta_{1} \leqq \pi$ and $0 \leqq \theta_{2}$ $\leqq 2 \pi$. In fact, we have from the equality in (1),

$$
(4 \pi)^{\frac{3}{2}} r^{K^{3}}=2 \sqrt{ } \pi r \int_{0}^{2 \pi} \int_{0}^{\pi}\left(\frac{r^{-2}}{r^{2} \sin \phi_{1} d \phi_{1} d \phi_{2}}\right)^{\frac{3}{2}} r^{2} \sin \theta_{1} d \theta_{1} d \theta_{2},
$$

so that

$$
\int_{0}^{2 \pi} \int_{0}^{\pi}\left(\sqrt{\frac{\sin \phi_{1} d \phi_{1} d \phi_{2}}{\sin \theta_{1} d \theta_{1} d \theta_{2}}}-1\right) \cdot \sin \phi_{1} d \phi_{1} d \phi_{2}=0
$$

whence follows that for all $0<r<1$

$$
\frac{\sin \phi_{1} d \phi_{1} d \phi_{2}}{\sin \theta_{1} d \theta_{1} d \theta_{2}}=1
$$

This implies that the area element at $\left(r, \theta_{1}, \theta_{2}\right)$ on $|x|=r$ is equal to that at $\left(r, \phi_{1}, \phi_{2}\right)$ on $|y|=r$ corresponding to $\left(r^{\frac{1}{K}}, \phi_{1}, \phi_{2}\right)$ on the image $|y|=r^{1}$ of $|x|=r$ by an extremal mapping $y=y(x)$. Therefore $\theta_{1}=\phi_{1}$, and accordingly $d \phi_{2}=d \theta_{2}$, so that $\phi_{2}=\theta_{2}+c, c$ being any real constant. Consequently, it has been shown that any extremal mapping is nothing but $y=y\left(x\left(r, \theta_{1}, \theta_{2}\right)\right)$ mentioned at the latter half of the theorem. The converse holds obviously. Our proof is completed.

7. The extremal mapping which induces the equality in the estimate obtained in Theorem 2 is uniquely determined except for rotations of the sphere $|x|<1$. If we exclude from the assumption of Theorem 2 the statement " $y(x)$ maps each radius of $|x|<1$ onto a curve which is normal to the image of each surface $|x|=r(<1)$ ", it remains indistinct whether the uniqueness of the extremal mapping in the above sense holds or not. However, we can show in general the following

PROPOSITION 1. Let $y=y(x)$ be any K-quasiconformal mapping of the $n$-dimensional sphere $|x|=\sqrt{x_{1}^{2}+x_{2}^{2}+\cdots+x_{n}^{2}}<1 \quad(n \geqq 3)$ into another such $|y|<1$ with $y(0)=0$. Then there holds 


$$
\liminf _{x \rightarrow 0}\left|y(x)_{\mid}\right||x|^{-\frac{1}{K}} \leqq 1,
$$

where the equality holds for such a mapping $y=y\left(x\left(r, \theta_{1}, \theta_{2}, \ldots, \theta_{n-1}\right)\right)$ as $y_{1}=r^{-\frac{1}{K}} \cos \theta_{1}, y_{2}=r^{-\frac{1}{K}} \sin \theta_{1} \cos \theta_{2}, y_{3}=r^{\frac{1}{K}} \sin \theta_{1} \sin \theta_{2} \cos \theta_{3}, \ldots, y_{n-1}=r^{\frac{1}{K}}$ $\sin \theta_{1} \sin \theta_{2} \sin \theta_{3} \cdots \sin \theta_{n-2} \cos \left(\theta_{n-1}+c\right), y_{n}=r^{K} \sin \theta_{1} \sin \theta_{2} \sin \theta_{3} \cdots \sin \theta_{n-2}$ $\sin \left(\theta_{n-1}+c\right)$ with any real constant $c$.

Proof. Denote by $m(r)$ the shortest distance from $y=0$ to the image of $|x|=r$ under $y=y(x)$. Then the image $y(r<|x|<1)$ of the ring $r<|x|<1$ under $y=y(x)$ is contained in the ring $m(r)<|y|<1$, so it separates the boundary components of the ring $m(r)<|y|<1$. Hence it follows from the monotoneity property of the modulus that

$$
\bmod y(r<|x|<1) \leqq \log \frac{1}{m(r)} .
$$

On the other hand, we get by the modulus condition,

$$
\frac{1}{K} \log \frac{1}{r} \leqq \bmod y(r<|x|<1) .
$$

Hence $m(r) / r^{1} \leqq 1$, so that

$$
\liminf _{x \rightarrow 0}|y(x)| /|x|^{-\frac{1}{K}}=\operatorname{lin}_{r \rightarrow 0} \inf m(r) / r^{\frac{1}{K}} \leqq 1 .
$$

Considering the inverse of a mapping in Proposition 1, we have

COROLlary 3. Let $y=y(x)$ be any Krquasiconformal mapping of a domain in $|x|=\sqrt{x_{1}^{2}+x_{2}^{2}+\cdots+x_{n}^{2}}<1$ onto $|y|<1$ with $y(0)=0$. Then there holds

$$
\lim \sup _{x \rightarrow 0}|y(x)| /|x|^{K} \geqq 1,
$$

where the equality holds for such a mapping $y=y\left(x\left(r, \theta_{1}, \ldots, \theta_{n-1}\right)\right)$ as $y_{1}$ $=r^{K} \cos \theta_{1}, y_{2}=r^{K} \sin \theta_{1} \cos \theta_{2}, y_{3}=r^{K} \sin \theta_{1} \sin \theta_{2} \cos \theta_{3}, \ldots, y_{n-1}=r^{K} \sin \theta_{1} \sin \theta_{2}$ $\sin \theta_{3} \cdots \sin \theta_{n-2} \cos \left(\theta_{n-1}+c\right), y_{n}=r^{K} \sin \theta_{1} \sin \theta_{2} \sin \theta_{3} \cdots \sin \theta_{n-2} \sin \left(\theta_{n-1}+c\right)$ with any real constant $c$.

Remark. It can be easily verified from our above proof that under the same assumption as in Theorem 2 or Proposition 1 , it holds for all $\alpha \leqq \frac{1}{K}$.

$$
\lim _{x \rightarrow 0} \inf |y(x)| /|x|^{\alpha} \leqq 1
$$


and, under the same assumption as in Corollary 3 , it holds for all $\alpha \geqq K$,

$$
\limsup _{x \rightarrow 0}|y(x)| /|x|^{\alpha} \geqq 1
$$

By the above results, we can enunciate in particular the following

COROLlary 4. Let $y=y(x)$ be any K-quasiconformal mapping of $|x|<1$ onto $|y|<1$ satisfying $y(0)=0$. Then for $\alpha \geqq K$,

$$
\liminf _{x \rightarrow 0}|y(x)| /|x|^{\frac{1}{\alpha}} \leqq 1 \leqq \lim _{x \rightarrow 0} \sup |y(x)| /|x|^{\alpha}
$$

Remark. If we exclude the restriction that the origin is carried into itself and each sphere is the unit one from the assumptions in Theorems 1,2 and their corollaries, the corresponding results to them can be obtained respectively by means of them and auxiliary Moebius transformations, which will be noted down elsewhere.

8. We denote by ' $\varsigma_{\alpha}$ the family of all $K$-quasiconformal mappings of $|x|<1$ into $|y|<\infty$ such that $y(0)=0$ and $\lim _{x \rightarrow 0}|y(x)| /|x|^{\alpha}=1, \alpha$ being real. Before we consider the distortion of mappings belonging to $\Xi_{\alpha}$, we shall establish the following general theorem indicating the range of such an $\alpha$ as $\varsigma_{\alpha}$ is empty.

TheOREM 3. Let $y=y(x)$ be a K-quasiconformal mapping of $|x|<1$ (into the Moebius 3-space). Assume that $y(0)=0$ and that the positive finite $\lim _{x \rightarrow 0}$ $|y(x)| /|x|^{\alpha}$ exists for a real number $\alpha$. Then $1 / K \leqq \alpha \leqq K$.

First we prepare for the proof of it.

Lemma 5. The sphere $|x|<1$ cannot be mapped onto $|y|<\infty$ by any $K$ quasicoformal mapping.

Proof. For completeness, we give a proof based essentially on the classical method due to $O$. Teichmüller.

Suppose, on the contrary, that $|x|<1$ can be mapped onto $|y|<\infty$ by a $K$. quasiconformal mapping $y=y(x)$. Then we can choose a suitable positive number $r_{1}(<1)$ such that the image of $|x| \leqq r_{1}$ by $y=y(x)$ contains the origin $y=0$. Put $\max _{|x|=r_{1}}|y(x)|=M$. Then there exists a real number $r_{2}\left(>r_{1}\right)$ such that $\min _{|x| \geqq r_{2}}|y(x)| \geqq M e^{x}$ for any positive number $X$. Since the ring $M<|y|<M e^{x}$ separates the boundary components of the image $y\left(r_{1}<|x|<r\right)$ of $r_{1}<|x|<r$ 
by $y=y(x)$, where $r_{2} \leqq r<1$, the monotoneity property of the modulus and the modulus condition imply

$$
K \log \frac{r}{r_{1}} \geqq \bmod y\left(r_{1}<|x|<r\right) \geqq \log \frac{M e^{x}}{M}=X,
$$

which is a contradiction, since $X$ can be chosen arbitrarily large.

Remark. It is noted by Lemma 5 that the complement $\mathscr{C} y(|x|<1)$, in the Moebius space, of the range of values taken by an arbitary $K$-quasiconformal mapping $y=y(x)$ of $|x|<1$ cannot degenerate into the point at infinity, and is also noted by using a suitable auxiliary Moebius transformation if necessary that it cannot reduce to the single finite point. Therefore, $\mathscr{C} y(|x|<1)$ is a (bounded or unbounded) continuum including at least two points.

Proposition 2. (An extension of Lemma 1). Suppose that $R$ is a ring in the Morbius space whose $C_{0}$ is bounded and contains the closed sphere $|x| \leqq a$ and whose another $C_{1}$ contains at least two points $x_{p}$ and $x_{q}$ satisfying $\left|x_{p}-x_{q}\right|$ $\leqq\left|x_{q}\right|-a$. Then there holds

$$
\bmod R \leqq \log \Phi_{3}\left(\frac{\left|x_{p}\right| \cdot\left|x_{a}\right|}{a\left|x_{p}-x_{q}\right|}\right)
$$

In particular case where $x_{q}$ is the point at infinity, this reduces to Lemma 1 .

Proof. Take the plane passing through three points the origin, $x_{p}$ and $x_{q}$ as the coordinate $\left(x_{1}, x_{2}\right)$-plane, and choose the oriented ray issuing from the origin and passing through $x_{q}$ as the positive $x_{1}$-axis, and introduce a system of cartesian coordinates $x_{1}, x_{2}, x_{3}$. We denote the coordinates of $x_{p}$ and $x_{q}$ respectively by $\left(p_{1}, p_{2}, 0\right)$ and $(q, 0,0)$.

Now, we will construct a transformation which carries $|x| \leqq a$ into itself and the point $x_{q}(q, 0,0)$ into the point at infinity. After some elementary computations, we can see that such a transformation is a composite mapping $y=y(x)$ of the following

$$
\begin{aligned}
& q-x_{1}=X_{1}, \quad x_{2}=X_{2}, \quad x_{3}=X_{3}, \\
& Y_{j}=\frac{\left(q^{2}-a^{2}\right) X_{j}}{X_{1}^{2}+X_{2}^{2}+X_{3}^{2}} \quad(j=1,2,3), \\
& y_{1}+q=Y_{1}, \quad y_{2}=Y_{2}, \quad y_{3}=Y_{3},
\end{aligned}
$$

which is a Moebius transformation and so 1-quasiconformal mapping. Hence 
we have $\bmod R=\bmod R^{\prime}, R^{\prime}$ being the image of $R$ by $y=y(x)$.

Denote by $y_{p}=y_{p}\left(p_{1}^{\prime}, p_{2}^{\prime}, p_{3}^{\prime}\right)$ the image of the point $x_{p}$ under the above composite mapping $y=y(x)$. Then it lies on the Gaussian $\left(y_{1}, y_{2}\right)$-plane and

$$
p_{1}^{\prime}=\frac{q p_{1}-a^{2}}{\left(q-p_{1}\right)-i p_{2}}, \quad p_{2}^{\prime}=\frac{q p_{2}}{\left(q-p_{1}\right)-i p_{2}}, \quad\left(p_{3}^{\prime}=0\right),
$$

hence it holds that

$$
\begin{aligned}
\left|y_{p}\right| & =\sqrt{\left(q p_{1}-a^{2}\right)^{2}+\left(q p_{2}\right)^{2}} / \sqrt{\left(q-p_{1}\right)^{2}+p_{2}^{2}} \\
& =\sqrt{\left(p_{1}^{2}+p_{2}^{2}\right) q^{2}-a^{2}\left(2 p_{1} q-a^{2}\right)} / \sqrt{\left(q-p_{2}\right)^{2}+p_{2}^{2}} \\
& \leqq \sqrt{\left(p_{1}^{2}+p_{2}^{2}\right) q^{2}} / \sqrt{\left(q-p_{1}\right)^{2}+p_{2}^{2}}=\left|x_{p}\right|\left|x_{q}\right| /\left|x_{p}-x_{q}\right| .
\end{aligned}
$$

The complement of the image $R^{\prime}$ of $R$ consists of two components $C_{0}^{\prime}$ and $C_{1}^{\prime}$ such that $C_{0}^{\prime}$ contains the closed sphere $|y| \leqq a$ and $C_{1}^{\prime}$ is unbounded and contains the point $y_{p}$. Consequently, by Lemma 1 we have

$$
\bmod R^{\prime} \leqq \log \varpi_{3}\left(\frac{\left|x_{p}\right|\left|x_{q}\right|}{a\left|x_{p}-x_{q}\right|}\right)
$$

Proof of Theorem 3. We let $y_{p}$ denote a point at the shortest distance from the origin to the complement of the image domain of $|x|<1$ under an arbitrary mapping $y=y(x)$ satisfying the condition of Theorem 3 . Then, by Lemma $5, y_{p}$ cannot be the point at infinity. Now if we set $\lim _{x \rightarrow 0}|y(x)| /|x|^{\alpha}=d$, then corresponding to $y_{p}$ and any positive number $\varepsilon(<d)$, there exists a positive number $\delta$ such that

$$
(d-\varepsilon)|x|^{\alpha}<|y(x)|<(d+\varepsilon)|x|^{\alpha}<\left|y_{p}\right|
$$

for $0<|x|<\delta$. Denote by $R$ the concentric spherical ring $r<|x|<1$, where $0<$ $r<\delta$, and by $y(R)$ the image of $R$ under $y=y(x)$. It is easily seen that

$$
\log \frac{\left|y_{p}\right|}{(d+\varepsilon) r^{\alpha}} \leqq \bmod y(R)
$$

On the other hand, we find in view of Remark after Lemma 5 that the complement of the image $y(R)$ consists of two components $C_{0}, C_{1}$, where $C_{0}$ contains the sphere $|y| \leqq(d-\varepsilon) r^{\alpha}$ and $C_{1}$ is a continuum including at least $y_{p}$ and $y_{q}$ such that $0<\left|y_{p}-y_{q}\right| \leqq\left|y_{q}\right|-(d-\varepsilon) \delta^{\alpha}$. Thus by Proposition 2,

$$
\bmod y(R) \leqq \log \emptyset_{3}\left(\frac{\left|y_{p}\right|\left|y_{q}\right|}{(d-\varepsilon) r^{\alpha}\left|y_{p}-y_{p}\right|}\right) .
$$


Since $\Phi_{3}(a) \leqq \lambda a$ for $a>1$ as is stated in $\S 2$, we get

$$
\bmod y(R) \leqq \log \frac{\lambda\left|y_{p}\right|\left|y_{q}\right|}{(d-\varepsilon) r^{\alpha}\left|y_{p}-y_{q}\right|}
$$

Furthermore, it holds by the modulus condition that

$$
\frac{1}{K} \log \frac{1}{r} \leqq \bmod y(R) \leqq K \log \frac{1}{r} .
$$

Form the above (5), (6) and (7), we obtain the following two relations:

$$
\begin{gathered}
\log \frac{\left|y_{p}\right|}{(d+\varepsilon) r^{\alpha}} \leqq K \log \frac{1}{r}, \\
\frac{1}{K} \log \frac{1}{r} \leqq \log \frac{\lambda\left|y_{p}\right|\left|y_{q}\right|}{(d-\varepsilon) r^{\alpha}\left|y_{p}-y_{q}\right|} .
\end{gathered}
$$

Here, by making $r \rightarrow 0$, it can be deduced that $\alpha \leqq K$ and $\frac{1}{K} \leqq \alpha$, which is the assertion.

Theorem 3 implies that the family $\Theta_{\alpha}$ is empty for $\alpha<\frac{1}{K}$ or $\alpha>K$. Moreover, it will be shown in $\S \mathbf{1 0}$ and $\S \mathbf{1 1}$ that $\Im_{\alpha}$ is not empty for $\frac{1}{K} \leqq \alpha \leqq K$.

9. Suppose that $w=w(z)=u(s, t)+i v(s, t)$ is a certain plane $K$-quasiconformal mapping of a symmetric domain $D_{2}$ with respect to the $s$-axis in the $z$-plane onto a domain $\Delta_{2}$ with the same property in the $w$-plane. We introduce a system of cartesian coordinates $x_{1}, x_{2}, x_{3}$ such that the $x_{1}$-axis and $x_{2}$-axis coincide with the $s$-axis and $t$-axis of the $z$-plane respectively, and denote by $D_{3}$ the space domain obtained by revolving $D_{2}$ about the $x_{1}$-axis. Following the same procedure for $u$ - and $v$-axes and the symmetric domain $\Delta_{2}$, we have $y_{1^{-}}, y_{2^{-}}$and $y_{3}$-axes and the space domain $\Delta_{3}$ of revolution about the $y_{1}$-axis. Then, the certain mapping $y=y(x)$ of $D_{3}$ onto $\Delta_{3}$ associated with $w=w(z)$ can be defined. In the next place, we treat with a relation between some mappings $w=w(z)$ and $y=y(x)$ of this kind.

PROPOSITION 3. Suppose that $w=w(z)=f(r) e^{i \theta}\left(w=u+i v, z=s+i t=r e^{i \theta}\right)$ is a $K$-quasiconformal mapping of $|z|<1$ in the classical sense. Then, $y=$ $y(x(r, \theta, \varphi)): y_{1}=f(r) \cos \theta, y_{2}=f(r) \sin \theta \cos \varphi, y_{3}=f(r) \sin \theta \sin . \varphi$ is also a K-quasiconformal mapping of $|x|=\sqrt{x_{1}^{2}+x_{2}^{2}+x_{3}^{2}}<1$ in the classical sense.

Proof. We have easily 


$$
|J(z)|=\left|\frac{\partial(u, v)}{\partial(s, t)}\right|=\left|\frac{\partial(u, v)}{\partial(r, \theta)} / \frac{\partial(s, t)}{\partial(r, \theta)}\right|=\frac{\left|f^{\prime}(r) f(r)\right|}{r} .
$$

Let $\left(w^{\prime}(\dot{z})\right)_{l, m}$ denote the directional derivative of $w(z)$ in the direction whose direction cosines are $l, m$. A simple calculation gives

$$
\begin{aligned}
\left|\left(w^{\prime}(z)\right)_{l, m}\right|^{2} & =\left|\frac{\partial w}{\partial s} l+\frac{\partial w}{\partial t} m\right|^{2} \\
& =\left\{f^{\prime}(r)^{2}-\left(\frac{f(r)}{r}\right)^{2}\right\}(l \cos \theta+m \sin \theta)^{2}+\left(\frac{f(r)}{r}\right)^{2} .
\end{aligned}
$$

Here, $l \cos \theta+m \sin \theta$ denotes the cosine of the angle between the ray from $z$ with its direction cosines $l, m$ and the ray passing through $z$ from the origin. Therefore it holds that $0 \leqq(l \cos \theta+m \sin \theta)^{2} \leqq 1$. Hence we have

$$
\max \left|\left(w^{\prime}(z)\right)_{l, m}\right|^{2}=f^{\prime}(r)^{2} \text { or }\left(\frac{f(r)}{r}\right)^{2}
$$

if $\left|f^{\prime}(r)\right| \geqq|f(r)| / r$ or $\left|f^{\prime}(r)\right| \leqq|f(r)| / r$, respectively, so that

$$
\frac{\max \left|\left(w^{\prime}(z)\right)_{l, m}\right|^{2}}{|J(z)|}=\frac{r\left|f^{\prime}(r)\right|}{|f(r)|} \text { or } \frac{|f(r)|}{r\left|f^{\prime}(r)\right|}
$$

Hence our assumption yields that

$$
\max \left(\frac{r\left|f^{\prime}(r)\right|}{|f(r)|} \cdot \frac{|f(r)|}{r\left|f^{\prime}(r)\right|}\right) \leqq K
$$

On the other hand, we have similarly

$$
\begin{aligned}
|J(x)| & =\left|\frac{\partial\left(y_{1}, y_{2}, y_{3}\right)}{\partial\left(x_{1}, x_{2}, x_{3}\right)}\right|=\left|\frac{\partial\left(y_{1}, y_{2}, y_{3}\right)}{\partial(r, \theta, \varphi)} / \frac{\partial\left(x_{1}, x_{2}, x_{3}\right)}{\partial(r, \theta, \varphi)}\right| \\
& =\left|f^{\prime}(r)\right| \cdot f(r)^{2} / r^{2} .
\end{aligned}
$$

Denote by $\left(y^{\prime}(x)_{l, m, n}\right.$ the directional derivative of $y(x)$ in the direction whose direction cosines are $l, m, n$. Then the square of its absolute value is expressed as follows :

$$
\begin{aligned}
& \left|\left(y^{\prime}(x)\right)_{l, m, n}\right|^{2}=\left(\frac{\partial y_{1}}{\partial x_{1}} l+\frac{\partial y_{1}}{\partial x_{2}} m+\frac{\partial y_{1}}{\partial x_{3}} n\right)^{2} \\
& \quad+\left(\frac{\partial y_{2}}{\partial x_{1}} l+\frac{\partial y_{2}}{\partial x_{2}} m+\frac{\partial y_{2}}{\partial x_{3}} n\right)^{2}+\left(\frac{\partial y_{3}}{\partial x_{1}} l+\frac{\partial y_{3}}{\partial x_{2}} m+\frac{\partial y_{3}}{\partial x_{3}} n\right)^{2} .
\end{aligned}
$$

After some elementary computations, we obtain

$$
\frac{\partial y_{1}}{\partial x_{1}}=\left(f^{\prime}(r)-\frac{f(r)}{r}\right) \cos ^{2} \theta+\frac{f(r)}{r},
$$




$$
\begin{aligned}
& \frac{\partial y_{2}}{\partial x_{2}}=\left(f^{\prime}(r)-\frac{f(r)}{r}\right) \sin ^{2} \theta \cos ^{2} \varphi+\frac{f(r)}{r}, \\
& \frac{\partial y_{3}}{\partial x_{3}}=\left(f^{\prime}(r)-\frac{f(r)}{r}\right) \sin ^{2} \theta \sin ^{2} \varphi+\frac{f(r)}{r}, \\
& \frac{\partial y_{1}}{\partial x_{2}}=-\frac{\partial y_{2}}{\partial x_{1}}=\left(f^{\prime}(r)-\frac{f(r)}{r}\right) \sin \theta \cos \theta \cos \varphi, \\
& \frac{\partial y_{1}}{\partial x_{3}}=\frac{\partial y_{3}}{\partial x_{1}}=\left(f^{\prime}(r)-\frac{f(r)}{r}\right) \sin \theta \cos \theta \sin \varphi, \\
& \frac{\partial y_{2}}{\partial x_{3}}=\frac{\partial y_{3}}{\partial x_{2}}=\left(f^{\prime}(r)-\frac{f(r)}{r}\right) \sin ^{2} \theta \sin \varphi \cos \varphi .
\end{aligned}
$$

Inserting these in the above expression, we have

$$
\begin{gathered}
\left|\left(y^{\prime}(x)\right)_{l, m, n}\right|^{2}=\left\{f^{\prime}(r)^{2}-\left(\frac{f(r)}{r}\right)^{2}\right\}(l \cos \theta+m \sin \theta \cos \varphi+n \sin \theta \sin \varphi)^{2} \\
+\left(\frac{f(r)}{r}\right)^{2} .
\end{gathered}
$$

Since $l \cos \theta+m \sin \theta \cos \varphi+n \sin \theta \sin \varphi$ means the cosine of the angle between the ray from $x$ with its direction cosines $l, m, n$ and the ray passing through $x$ from the origin, it holds $0 \leqq(l \cos \theta+m \sin \theta \cos \varphi+n \sin \theta \sin \varphi)^{2} \leqq 1$. Therefore, if $f^{\prime}(r)^{2} \geqq(f(r) / r)^{2}$ (or $\left.f^{\prime}(r)^{2} \leqq(f(r) / r)^{2}\right)$, then

$$
\begin{aligned}
& \left.\max \left|\left(y^{\prime}(x)\right)_{l, m, n}\right|^{2}=f^{\prime}(r)^{2} \quad \text { (or }(f(r) / r)^{2}\right), \\
& \left.\min \left|\left(y^{\prime}(x)\right)_{l, m, n}\right|^{2}=(f(r) / r)^{2} \quad \text { (or } f^{\prime}(r)^{2}\right) \text {. }
\end{aligned}
$$

Thus we find that

$$
\begin{aligned}
& \frac{\max _{l, m, n}\left|\left(y^{\prime}(x)\right)_{l, m, n}\right|^{3}}{|J(x)|}=\left(\frac{r f^{\prime}(r)}{f(r)}\right)^{2} \text {, and } \frac{|J(x)|}{\min _{l, m, n}\left|\left(y^{\prime}(x)\right) l, m, n\right|^{3}}=\frac{r\left|f^{\prime}(r)\right|}{|f(r)|}, \\
& \left(\text { or } \frac{\max _{1, m, n}\left|\left(y^{\prime}(x)\right)_{l, m, n}\right|^{3}}{|J(x)|}=\frac{|f(r)|}{r\left|f^{\prime}(r)\right|} \text { and } \min _{i, m, n} \frac{|J(x)|}{\left|\left(y^{\prime}(x)\right)_{l, m, n}\right|^{3}}=\left(\frac{f(r)}{r f^{\prime}(r)}\right)^{2}\right) \text {. }
\end{aligned}
$$

Recalling here the inequality (8), we have

$$
\max \left(\frac{\max \left|\left(y^{\prime}(x)\right)_{l, m, n}\right|^{3}}{|J(x)|}, \frac{|J(x)|}{\min \left|\left(y^{\prime}(x)\right)_{l, m, n}\right|^{3}}\right) \leqq K^{2} .
$$

Moreover, since $w=w(z)$ is a continuously differentiable homeomorphism of $|z|<1$, we see that $y=y(x(r, \theta, \varphi))$ has also the same property in $|x|<1$. Thus $y=y(x)$ is a $K$-quasiconformal mapping of $|x|<1$ in the classical sense.

10. Now, we consider the family $\varsigma_{\alpha}$ of all $K$-quasiconformal mappings of 
$|x|<1$ into the Euclidean 3-space $|y|<\infty$ such that $y(0)=0$ and $\lim _{x \rightarrow 0}|y(x)| /|x|^{\alpha}$ $=1, \alpha$ being real, and discuss about the cases where $\inf _{y \in \widetilde{\Phi} \alpha} \min _{|x|=r}|y(x)|$ is positive and $\sup _{y \in \mathscr{E}_{\alpha}} \max _{|x|=r}|y(x)|$ is finite and where there exists the so-called Koebe's constant for $\mathfrak{\subseteq}_{\alpha}$ and $\mathfrak{S}_{\alpha}$ is a normal family.

THEOREM 4. The infimum of $\min _{0<|x|=r \leqq 1}|y(x)|$ for all $y(x) \in \Im_{\alpha}$ is positive if and only if $\alpha=\frac{1}{K}$.

Proof. (i) The case $\alpha=\frac{1}{K}$. The proof in this case proceeds similarly to that due to Gehring. Let $y=y(x)$ be an arbitrary mapping belonging to $\bigodot_{\alpha}$, and denote the image of the ring $r^{\prime}<|x|<r$ for $0<r^{\prime}<r<1$ under $y=y(x)$ by $y\left(r^{\prime}<|x|<r\right)$. Then the complement of $y\left(r^{\prime}<|x|<r\right)$ has two components, one of which is bounded and contains the sphere $|y| \leqq \min _{|x|=r^{\prime}}|y(x)|$ and the other of which is unbounded. Thus we have by the modulus condition and Lemma 1 ,

$$
\frac{1}{K} \log \frac{r}{r^{\prime}} \leqq \bmod y\left(r^{\prime}<|x|<r\right) \leqq \log \emptyset_{3}\left(\frac{\min _{|x|=r}|y(x)|}{\min _{|x|=r^{\prime}}|y(x)|}\right) .
$$

In view of the estimate $\Phi_{3}(a) \leqq \lambda a$ for $a>1$ stated in $\S 2$, we have

$$
\left(\frac{r}{r^{\prime}}\right)^{-\frac{1}{K}} \leqq \frac{\lambda \min _{i x \mid=r}|y(x)|}{\min _{|x|=r^{\prime}}|y(x)|}
$$

so that

$$
\min _{0<|x|=r<1}|y(x)| \geqq \frac{r^{\dddot{K}} \min _{|x|=r^{\prime}}|y(x)|}{\lambda r^{\prime \frac{1}{K}}}
$$

Letting $r^{\prime} \rightarrow 0$, we have for all $y(x) \in S_{K}^{1}$,

$$
\min _{0<|x|=r<1}|y(x)| \geqq r^{1} / \lambda,
$$

which is also valid for $r=1$.

(ii) The case $\frac{1}{K}<\alpha \leqq 1$. Consider $y=y(x(r, \theta, \varphi), n)$ :

$$
y_{1}=f_{n}(r) \cos \theta, y_{2}=f_{n}(r) \sin \theta \cos \varphi, y_{3}=f_{n}(r) \sin \theta \sin \varphi,
$$

where $f_{n}(r)=r^{\alpha}\left\{1-\left(1-\frac{1}{n}\right) r^{\frac{\alpha_{K}-1}{K(n-1)}}\right\}$. Then it is a $K$-quasiconformal mapping 
of $|x|<1$ in the classical sense by Proposition 3, because it is proved in [5] that $w=w(z, n)=\dot{f}_{n}(r) e^{i \theta}\left(z=r e^{i \theta}\right)$ is a $K$-quasiconformal mapping of $|z|<1$ in the classical sense. Obviously, $y(0, n)=0$ and $\lim _{x \rightarrow 0}|y(x, n)| /|x|^{\alpha}=1$, and hence $y=y(x, n) \in \Im_{\alpha}$. Since $y=y(x, n)$ maps $|x|<1$ onto $|y|<\frac{1}{n}$, we obtain $\inf _{y \in \mathscr{E}_{\alpha}} \min _{0<|x|=r \cong 1}|y(x)|=0$ by making $n \rightarrow \infty$.

(iii) The case $1<\alpha \leqq K$. Take $y=y(x(r, \theta, \varphi), n)$ :

$$
\begin{gathered}
y_{1}=r^{\alpha} \cos \theta, y_{2}=r^{\alpha} \sin \theta \cos \varphi, y_{3}=r^{\alpha} \sin \theta \sin \varphi \text { for }|x|<\frac{1}{n}, \\
y_{1}=r\left(\frac{1}{n}\right)^{\alpha-1} \cos \theta, y_{2}=r\left(\frac{1}{n}\right)^{\alpha-1} \sin \theta \cos \varphi, y_{3}=r\left(\frac{1}{n}\right)^{\alpha-1} \sin \theta \sin \varphi \\
\text { for } 1 / n \leqq|x|<1 .
\end{gathered}
$$

It is easy to see that this mapping $y=y(x, n)$ is an $\alpha$-and so $K$-quasiconformal mapping of $|x|<1$ and belongs to $\Xi_{\alpha}$. We have $|y(x, n)|=r^{\alpha}<\left(\frac{1}{n}\right)^{\alpha} \rightarrow .0(n$ $\rightarrow \infty)$ for $|x|=r<\frac{1}{n}$, and $|y(x, n)|=r\left(\frac{1}{n}\right)^{\alpha-1}<\left(\frac{1}{n}\right)^{\alpha-1} \rightarrow 0(n \rightarrow \infty)$ for $\frac{1}{n} \leqq$ $|x|=r<1$. Thus we get $\inf _{y \in \coprod_{\alpha}} \min _{0<|x|=r \leqq 1}|y(x)|=0$.

Since $\bigodot_{\alpha}$ is empty for $\alpha>K$ or $\alpha<\frac{1}{K}$ by Theorem 3, our proof is completed.

In particular, we have the following

Corollary 5. For the family $\bigodot_{\alpha}$, there exists the so.called Koebe's constant if and only if $\alpha=\frac{1}{K}$. Further, if $\alpha \neq \frac{1}{K}$. then there exists no Koebe's constant even for the subfamily of $\mathfrak{S}_{\alpha}$ each mapping of which is K-quasiconformal in the classical sense.

Remark. Letting $r \rightarrow 1$ in the estimate (9) showed in the above proof, we find immediately for any $K$-quasiconformal mapping of $|x|<1$ into $|y|<\infty$ with $y(0)=0$ that

$$
\min _{|x|=1}|y(x)| \geqq \frac{1}{\lambda} \limsup _{s \rightarrow 0} \frac{\min _{|x|=s}|y(x)|}{s^{\frac{1}{K}}},
$$

which is equivalent to the estimate (52) in Gehring [3] as is easily verified.

11. We can find the following facts in contrast with the results in $\S \mathbf{1 0}$.

THEOREM 5. The supremum of $\max _{0<|x|=r<1}|y(x)|$ for all $y(x) \in \mathcal{S}_{\alpha}$ is finite if and only if $\alpha=K$. 
Before proving the theorem, we prove the following lemma.

LeMm-A 6. The supremum of $\min _{0<|x|=r \leqq 1}|y(x)|$ for all $y(x) \in \S_{K}$ is not greater than $(\lambda r)^{K}$.

Proof: Let $y=y(x)$ be any mapping belonging to $\Im_{K}$, and put $\min _{0<|x|=r<1}|y(x)|$ $=m$. Then the inverse mapping $x=x(y)$ of $y=y(x)$ maps $|y|<m$ into $|x|<r$ $<1$. Let $R_{y}$ denote the sphere $|y|<m$ slit along the segment from the origin to the point $y$, and let $x\left(R_{y}\right)$ denote the image of $R_{y}$ under $x=x(y)$. Then the monotoneity property of the modulus and Lemma 1 yield

$$
\bmod R_{y}=\log \emptyset_{3}\left(\frac{m}{|y|}\right) \text { and } \bmod x\left(R_{y}\right) \leqq \log \Phi_{3}\left(\frac{r}{|x|}\right)
$$

Take the modulus condition $\frac{1}{K} \bmod R_{y} \leqq \bmod x\left(R_{y}\right)$ and the estimate $a \leqq \Phi_{3}(a)$ $\leqq \lambda a$ for $a>1$ into consideration. Then

$$
\frac{m}{|y|} \leqq \Phi_{3}\left(\frac{m}{|y|}\right) \leqq\left\{\Phi_{3}\left(\frac{r}{|x|}\right)\right\}^{K} \leqq\left(\frac{\lambda r}{|x|}\right)^{K},
$$

so that

$$
m \leqq(\lambda r)^{K}|y(x)| /|x|^{K}
$$

Letting $x \rightarrow 0$ here, we have

$$
\sup _{y \in \Theta_{k}} \min _{0<|x|=r<1}|y(x)| \leqq(\lambda r)^{K},
$$

which is also valid for $r=1$.

Proof of Theorem 5. (i) The case $\alpha=K$. Let $y(r<|x|<1)$ be the image of the ring $0<r<|x|<1$ by any $y=y(x) \in \varsigma_{\alpha}$. Then we have by the modulus condition and Lemma 2 ,

$$
\frac{1}{K} \log \frac{1}{r} \leqq \bmod y(r<|x|<1) \leqq \log \Psi_{3}\left(\frac{\min _{|x|=1}|y(x)|}{\max _{|x|=r}|y(x)|}\right) .
$$

Since $\Psi_{3}(a)$ is a non-decreasing function of $a$, we get

$$
\max _{0<|x|=r<1}|y(x)| \leqq \min _{|x|=1}|y(x)| / \Psi_{3}^{-1}\left\{\left(\frac{1}{r}\right)^{-\frac{1}{K}}\right\} .
$$

where $\Psi_{3}^{-1}(c)$ denotes the inverse function of $c=\Psi_{3}(a)$. In view of Lemma 6, we have for all $y(x) \in \bigodot_{\alpha}$, 


$$
\max _{0<|x|=r<1}|y(x)| \leqq \lambda^{K} / \Psi_{3}^{-1}\left\{\left(\frac{1}{r}\right)^{-\frac{1}{K}}\right\} .
$$

(ii) The case $1 \leqq \alpha<K$. Consider $y=y(x(r, \theta, \varphi), n)$ :

$$
y_{1}=f_{n}(r) \cos \theta, y_{2}=f_{n}(r) \sin \theta \cos \varphi, y_{3}=f_{n}(r) \sin \theta \sin \varphi,
$$

where $f_{n}(r)=r^{\alpha}\left\{1+(n-1) r^{\frac{\alpha}{n-1}}\right\}$ for $n>\frac{K}{K-\alpha}$. Since it is shown in [5] that $w=w(z, n)=f_{n}(r) e^{i \theta}\left(z=r e^{i \theta}\right)$ is a $K$-quasiconfomal mapping of $|z|<1$ in the classical sense, Proposition 3 implies that $y=y(x(r, \theta, \varphi), n)$ is also a $K$-quasiconformal mapping of $|x|<1$ in the classical sense. Evidently $y=y(x, n)$ satisfies the normalization $y(0, \mathrm{n})=0$ and $\lim _{x \rightarrow 0}|y(x, n)| /|x|^{\alpha}=1$, so that it belongs to $\bigodot_{\alpha}$. Furthermore $y=y(x, n)$ maps $|x|<1$ onto $|y|<n$ and

$$
\left|y\left(x\left(r, \frac{\pi}{2}, \frac{\pi}{2}\right), n\right)\right|=f_{n}(r)=r^{\alpha}\left\{1+(n-1) r^{\frac{\alpha}{n-1}}\right\} \rightarrow+\infty \quad(n \rightarrow \infty) .
$$

(iii) The case $1 / K \leqq \alpha<1$. Take $y=y(x(r, \theta, \varphi), n)$ as the mapping with the form mentioned in the case $1<\alpha \leqq K$ in the proof of Theorem 4 . Then, it is similarly verified that this mapping is $K$-quasiconformal in $|x|<1$ in the classical sense and satisfies our normalization at the origin, so that it belongs to $\varsigma_{\alpha}$. If we take $n$ such that $n>1 / r$ for a given $r(0<r<1)$, then we see that on $|x|=r$,

$$
|y(x, n)|=r\left(\frac{1}{n}\right)^{\alpha-1}=r n^{1-\alpha} \rightarrow+\infty \quad(n \rightarrow \infty) .
$$

Thus our theorem is established.

A family $\{y(x)\}$ of continuous vector-valued functions $y(x)$, defined in a domain $D$, is called a normal family if every infinite subfamily of $\{y(x)\}$ contains a sequence which converges uniformly on every compact subset of $D$. Then we have

Corollary 6. The family $\bigodot_{\alpha}$ is normal if and only if $\alpha=K$.

Proof. It is easily verified that the estimate $\max _{0<|x|=r<1}|y(x)| \leqq \lambda^{K} / \Psi_{3}^{-1}\left\{(1 / r)^{\frac{1}{K}}\right\}$ obtained in the case (i) in the proof for Theorem 5 is also valid for $0<|x|<r$ $<1$. Hence we have for all $x$ in the sphere $\left|x-x_{p}\right|<1-\sqrt{\left|x_{p}\right|}$ about each point $x_{p}$ in $|x|<1$ and all $y(x) \in \varsigma_{K}$,

$$
|y(x)| \leqq \lambda^{K} / \Psi_{3}^{-1}\left\{\left(\frac{1}{1-\sqrt{\left|x_{p}\right|}+\left|x_{p}\right|}\right)^{K}\right\},
$$


whose right hand side will be simply denoted by $M\left(x_{p}\right)$. Then, $Y(X)=y\left(x_{p}\right.$ $+X)-y\left(x_{p}\right)$ is $K$-quasiconformal with $Y(0)=0$ and $|Y(X)| \leqq 2 M\left(x_{p}\right)$ in $|X|<$ $1-\sqrt{\left|x_{p}\right|}$. Thus, we see by Theorem 1 ,

$$
\left\{\emptyset_{3}\left(\frac{1-\sqrt{\left|x_{p}\right|}}{|X|}\right)\right\}^{\kappa} \leqq \emptyset_{3}\left(\frac{2 M\left(x_{p}\right)}{|Y|}\right) .
$$

Using the estimate $a \leqq \Phi_{3}(a) \leqq \lambda a$ for $a>1$, we get

$$
\left(\frac{1-\sqrt{\left|x_{p}\right|}}{|X|}\right)^{\frac{1}{K}} \leqq \frac{2 \lambda M\left(x_{p}\right)}{|Y|},
$$

so that

$$
y\left(x_{p}+X\right)-\left.y\left(x_{p}\right)\left|\leqq \frac{2 \lambda M\left(x_{p}\right)}{\left(1-\sqrt{\left|x_{p}\right|}\right)^{1 / K}}\right| X\right|^{\left.\right|^{3}} .
$$

Hence, putting $x_{p}+X=x$, we have for $\left|x-x_{p}\right|<1-\sqrt{\left|x_{p}\right|}$,

$$
\left|y(x)-y\left(x_{p}\right)\right| \leqq \frac{2 \lambda M\left(x_{p}\right)}{\left(1-\sqrt{\left|x_{p}\right|}\right)^{1 / K}}\left|x-x_{p}\right|^{\stackrel{1}{\kappa}} .
$$

Further, put $\delta\left(x_{p}\right)=\min \left\{1-\sqrt{\left|x_{p}\right|,}\left(1-\sqrt{\left|x_{p}\right|}\right) \varepsilon^{K} /\left(2 \lambda M\left(x_{p}\right)\right)^{K}\right\}$ for an arbitrary positive number $\varepsilon$. Then it follows that

$$
\left|y(x)-y\left(x_{p}\right)\right|<\varepsilon
$$

for all $x$ in $\left|x-x_{p}\right|<\delta\left(x_{p}\right)$ and all $y(x) \in \Im_{K}$, which yields that the family $\varsigma_{K}$ is equicontinuous at each point of $|x|<1$. Hereafter, almost similarly to the proof for the familiar Ascoli-Arzelà's theorem, it can be proved that $\mathfrak{夭}_{K}$ is a normal family in $|x|<1$.

In the case $\alpha \neq K$, by using our examples mentioned in the cases (ii) and (iii) in the proof of Theorem 5, it is easy to show that the family $\S_{\alpha}$ cannot be normal.

\section{Correspondence under quasiconformal mappings}

12. Let $E$ be any compact set in the bounded domain $D$ in 3-space, and denote by $C(E)$ the logarithmic capacity of $E$, by $m_{0}(E)$ the (outer) logarithmic measure of $E$, by $C^{(\alpha)}(E)$ the capacity of order $\alpha$ of $E$ and by $m_{\alpha}(E)$ the (outer) $\alpha$-dimensional measure of $E$. Then, we have immediately the following space form of a result by A. Mori [7]:

THEOREM 6. Let $y=y(x)$ be a K-quasiconformal mapping of the bounded 
domain $D_{x}$ in 3-space onto another such domain, and let $E_{x}$ be a compact set in $D_{x}$ and $E_{y}$ its image by $y(x)$. Then it hold

$$
\begin{aligned}
& C\left(E_{y}\right) \leqq A\left\{C\left(E_{x}\right)\right\}^{\frac{1}{K}}, \\
& m_{0}\left(E_{y}\right) \leqq K m_{0}\left(E_{x}\right), \\
& C^{(\alpha)}\left(E_{y}\right) \leqq A\left\{C^{\left(\frac{\alpha}{K}\right)}\left(E_{x}\right)\right\}^{-\frac{1}{K}} \text { and } \\
& m_{\alpha}\left(E_{y}\right) \leqq A^{\alpha} m_{\underset{K}{\alpha}}^{\alpha}\left(E_{x}\right),
\end{aligned}
$$

where $0<\alpha \leqq 3$ and $A$ is such a constant independent of $y(x)$ as in the next Lemma 7 due to Gehring (cf. [3], Cor. 6).

Lemma 7. Let $y=y(x)$ be a K-quasiconformal mapping of a bounded domain $D_{x}$ onto another such domain, and $E_{x}$ be any compact set contained in $D_{x}$. Then, corresponding to $E_{x}$ and its image set $E_{y}$, there exists a constant $A$ independent of $y(x)$ such that

$$
\left|y^{(1)}-y^{(2)}\right| \leqq A\left|x^{(1)}-x^{(2)}\right|^{k}
$$

for all $x^{(1)}, x^{(2)}$ in $E_{x}$ and their images $y^{(1)}=y\left(x^{(1)}\right), y^{(2)}=y\left(x^{(2)}\right)$.

Proof of Theorem 6. It is well known that for any compact set $E, C(E)$ (resp. $C^{(\alpha)}(E)$ ) is equivalent to the transfinite diameter of $E$ (resp. of order $\alpha$ of $E$ ), whose definition is

$$
\begin{gathered}
\lim _{n \rightarrow \infty} \max _{x^{(1)}, \ldots, x^{(n)} \in E}\left(\begin{array}{l}
n \\
2
\end{array}\right) \sqrt{\prod_{j<k}^{1, \ldots, n}\left|x^{(j)}-x^{(k)}\right|} \\
\text { (resp. } \left.\lim _{n \rightarrow \infty} \max _{x^{(1)}, \ldots, x^{(n)} \in E}\left\{\left(\begin{array}{l}
n \\
2
\end{array}\right) / \sum_{j<k}^{1, \ldots, n} \frac{1}{\left|x^{(j)}-x^{(k)}\right|^{\alpha}}\right\}^{1 / \alpha}\right) .
\end{gathered}
$$

(Cf. Frostman's thesis or Ugaheri [10]). Thus, (10) and (12) are immediate consequences of these relations and Lemma 7.

(11) is proved as follows: Take a countable number of subset $U_{j}$ such that the diameter $\delta\left(U_{j}\right)<\varepsilon<1$ and $\sum_{j} U_{j} \supset E_{x}$, and put $V_{j}=y\left(U_{j}\right)$. Then, for any $0<\varepsilon^{\prime}<1$, it is noted by Lemma 7 that there exists a positive number $\varepsilon_{0}$ such that $\delta\left(V_{j}\right)<\varepsilon^{\prime}$ and $0<K|\log A| / \log \left(1 / \delta\left(U_{j}\right)\right)<\frac{1}{2}$ for all $0<\varepsilon \leqq \varepsilon_{0}$. Thus by making use of Lemma 7 again, we get

$$
\frac{1}{\log \frac{1}{\delta\left(V_{j}\right)}} \leqq \frac{1}{\left\{1-K|\log A| / \log \frac{1}{\delta\left(\bar{U}_{j}\right)}\right\} \log \frac{1}{\delta\left(U_{j}\right)^{1 / K}}}
$$




$$
\leqq K\left(1+2 K|\log A| / \log \frac{1}{\varepsilon}\right) / \log \frac{1}{\delta\left(U_{j}\right)},
$$

from which we have

$$
\begin{aligned}
m_{0}\left(E_{y}\right) & \leqq \lim _{\varepsilon \rightarrow 0} \inf _{\left(V_{j}\right\}} \sum_{j} \frac{1}{\log \frac{1}{\delta\left(V_{j}\right)}-} \\
& \leqq \lim _{\varepsilon \rightarrow 0} K\left(1+2 K|\log A| / \log \frac{1}{\varepsilon}\right) \inf _{\left(U_{j}\right)} \sum_{j} \frac{1}{\log \frac{1}{\delta\left(U_{j}\right)}}=K m_{0}\left(E_{x}\right) .
\end{aligned}
$$

Since (13) is shown similarly to the above, we shall omit here.

13. A domain $D$ is called a (space) Jordan domain if its boundary is homeomorphic to the spherical surface $|x|=1$. Then, the following fact can be verified by using the similar argument due to Väisälä ([12], §2) or Gehring ([3], §§ 22-24).

Lemma 8. Suppose that a part of the boundary of a space Jordan domain $D_{x}$ consists of a plane Jordan domain $G_{x}$. Then any K-quasiconformal mapping $y(x)$ of $D_{x}$ onto another Jordan domain $D_{y}$ can be extended to a homeomorphism of $D_{x}+G_{x}$. Suppose moreover that its homeomorphism maps $G_{x}$ onto another plane Jordan domain $G_{y}$. Then $y(x)$ can be extended as a K-quasiconformal mapping outside $D_{x}$ over $G_{x}$.

Such a mapping as extended by Lemma 8 are led to a $K$-quasiconformal one of a space domain including $G_{x}$ onto another such domain including $G_{y}$ which let $G_{x}$ correspond to $G_{y}$, and the correspondence between $G_{x}$ and $G_{y}$ by such a mapping induces a plane quasiconformal mapping being not always $K$ quasiconformal. For instance, $y=y(x): y_{1}=x_{1}, y_{2}=K^{3} x_{2}, y_{3}=K^{2} x_{3}$ is a $K$ quasiconformal mapping in space, and the correspondence between $\left(x_{1}, x_{2}\right)$. plane and $\left(y_{1}, y_{2}\right)$-plane induced by $y=y(x)$ is the plane quasiconformal mapping but not the plane $K$-quasiconformal one. However, since such a correspondence between $G_{x}$ and $G_{y}$ is absolutely continuous or measurable, it transforms any set of 2 -dimensional measure zero on $G_{x}$ into a set of the same measure on $G_{y}$.

Now we have the following from Theorem 6 .

Corollary 7. Let $y=y(x)$ be a K-quasiconformal mapping of a bounded domain $D_{x}$ in space onto another such $D_{y}$ such that $y=y(x)$ let a plane domain 
$G_{x}$ on the closure of $D_{x}$ correspond to a plane domain $G_{y}$ on the closure of $D_{y}$. For a compact set $E_{x}$ in $G_{x}$, if the logarithmic capacity, measure, capacity of order $\alpha$, and $\alpha$-dimensional measure of $E_{x}$ are equal to zero, then the logarithmic capacity, measure, capacity of order $K \alpha$, and Ka-dimensional measure of the image $E_{y}$ of $E_{x}$ by $y=y(x)$ are also equal to zero, respectively.

14. As is proved by Gehring [3], any $K$-quasiconformal mapping $y=y(x)$, defined in a space domain $\mathrm{D}$, is measurable, and hence it transforms any set of 3-dimensional measure zero in $D$ into a set of the same property. However, it is indistinct whether $y=y(x)$ transforms any set of $\alpha(0<\alpha<3)$-dimensional measure zero into a set of the same property. In the next place, we shall give a criterion for both some closed set $E_{x}$ in a Jordan domain $D$ and its image set under any $K$-quasiconformal mapping $y=y(x)$ of $D$ to be of the same $\alpha$-dimensional measure zero.

Let $E$ be a compact set in 3 -space and let its complement $\mathscr{C} E$ be a (connected) domain. A set $\left\{R_{n}^{3(j)}\right\}(j=1,2, \ldots, \nu(n)<\infty ; n=1,2, \ldots)$ of rings $R_{n}^{3(j)}$ will be called a system inducing an exhaustion of $\mathscr{C} E$ if it satisfies the following conditions:

(i) the closure $\overline{R_{n}^{3(j)}}$ of $R_{n}^{3(j)}$ is contained in $\mathscr{C} E$,

(ii) the one component $C_{0, n}^{3(j)}$ of the complement of $R_{n}^{3(j)}$ has at least one point common with $E$, and the other component $C_{1, n}^{3(j)}$ contains the point at infinity,

(iii) any point of $E$ is contained in a certain $C_{0, n}^{3(j)}$,

(iv) $R_{n}^{3(k)}$ lies in $C_{1, n}^{3(j)}$ if $k \neq j$,

(v) each $R_{n+1}^{3(k)}$ is contained in a certain $C_{0, n}^{3(j)}$, and

(vi) $\left\{\mathscr{C} E_{n}\right\}_{n=1}^{\infty}$ is an exhaustion of $\mathscr{C} E$, where

$$
\mathscr{C} E_{n}=\bigcap_{j=1}^{\nu(n)}\left(C_{1, n}^{3(j)} \cup R_{n}^{3(j)}\right)
$$

Denote by $\bmod P_{x, n}^{3(j)}=\log \mu_{x, n}^{3(j)}$ the modulus of $R_{x, n}^{3(j)}$, and put $\min _{1 \leqq j \leqq v(n)} \log \mu_{x, n}^{3(j)}$ $=\log \mu_{3, n}$. We can prove the following space analogue of our previous result.

THeOREM 7. Let $E_{x}$ be such $a$ set as $E$ stated above and $y=y(x)$ be any $K$-quasiconformal mapping of a Jordan domain $D$ including $E_{x}$. If there exists a system $\left\{R_{n}^{3(j)}\right\}(j=1,2, \ldots, \nu(n)<\infty ; n=1,2, \ldots)$ inducing an exhaustion of $\mathscr{C} E_{x}$ which satisfies 


$$
\limsup _{n \rightarrow \infty}\left\{\alpha \sum_{l=1}^{n} \log \Psi_{3}^{-1}\left(\mu_{3, l}^{\frac{1}{K}}\right)-\left(1-\frac{\alpha}{3}\right) \log \nu(n)\right\}=+\infty
$$

for some $\alpha$ such that $0<\alpha \leqq 3$, then $E_{x}$ and its image $E_{y}$ by $y=y(x)$ are of $\alpha$ dimensional measure zero.

Proof. First, take a point $x_{0, n}^{(j)}$ in $C_{0, n}^{3, j)}$ and put

$$
r_{x, n}^{(j)}=\max _{x \in r_{0, n}^{(j)}}\left|x-x_{0, n}^{(j)}\right|, \rho_{x, n}^{(j)}=\min _{x \in R_{1, n}^{(j)}}\left|x-x_{0, n}^{(j)}\right|,
$$

where $B_{0, n}^{(j)}$ and $B_{1, n}^{(j)}$ are the boundaries of $C_{0, n}^{3(j)}$ and $C_{1, n}^{3(j)}$, respectively.

Since there exists a number $N_{D}$ such that the sub-system of $\left\{R_{n}^{3(j)}\right\}$ for $n$ $\geqq N_{D}$ is contained in $D$, we can difine similarly $\mu^{3(j), n}, r_{y, n}^{3(j)}$ and $\rho_{y, n}^{3(j)}$ concerning the images $R_{y, n}^{\left.3^{\prime} j\right)}, y\left(B_{0, n}^{3(j)}\right)$ and $y\left(B_{1, n}^{3(j)}\right)$ of $R_{x, n}^{3(j)}, B_{0, n}^{3(j)}$ and $B_{1, n}^{3(j)}$ for $n \geqq N_{D}$, respectively. Evidently, the image set $\left\{R_{y, n}^{3(j)}\right\}$ of $\left\{R_{x, n}^{3(j)}\right\}\left(j=1,2, \ldots, \nu(n)<\infty ; n=N_{n}\right.$, $\left.N_{n}+1, \ldots\right)$ is a system inducing an exhaustion of the complement $\mathscr{C} E_{y}$. By the modulus condition and Lemma 2 , we have for $n \geqq N_{D}$,

$$
\frac{1}{K} \log \mu_{3, n} \leqq \log \mu_{y, n}^{3(j)} \leqq \log \Psi_{3}\left(\rho_{y, n}^{(j)} / r_{y, n}^{(j)}\right),
$$

or

$$
r_{y, n}^{(j)} \leqq \rho_{y, n}^{(j)} / \Psi_{3}^{-1}\left(\mu_{3, n}^{1 / K}\right)
$$

Similarly we have

$$
r_{x, n}^{(j)} \leqq \rho_{x, n}^{(j)} / \Psi_{3}^{-1}\left(\mu_{3, n}\right) .
$$

Starting from (14) and applying Hölder's inequality, we have for $n \geqq N_{D}$ and $0<\alpha \leqq 3$,

$$
\begin{aligned}
\sum_{j=1}^{\nu(n)}\left(\boldsymbol{r}_{y, n}^{(j)}\right)^{\alpha} & \leqq \frac{1}{\left\{\Psi_{3}^{-1}\left(\mu_{3, n}^{1 / K}\right)\right\}^{\alpha}} \sum_{j=1}^{\nu(n)}\left(\rho_{y, n}^{(j)}\right)^{\alpha} \\
& \leqq \frac{\nu(n)^{1-\alpha / 3}}{\left\{\Psi_{3}^{-1}\left(\mu_{3, n}^{1 / K}\right)\right\}^{\alpha}}\left\{\sum_{j=1}^{\nu(n)}\left(\rho_{y, n}^{(j)}\right)^{3}\right\}^{\alpha / 3} .
\end{aligned}
$$

Now, it is obvious that $\frac{4 \pi}{3} \sum_{j=1}^{\nu(n)}\left(\rho_{y, n}^{(j)}\right)^{3}$ is not greater than the sum of volumes bounded by $\bigcup_{j=1}^{\nu(n)} y\left(B_{1, n}^{(j)}\right)$ and that $\frac{4 \pi}{3} \sum_{k=1}^{\nu(n-1)}\left(r_{y, n-1}^{(k)}\right)$ is not less than the sum of volumes bounded by $\bigcup_{k=1}^{\nu(n-1)} y\left(B_{0, n}^{(k)}\right)$, and so

$$
\sum_{j=1}^{\nu(n)}\left(\rho_{y, n}^{(j)}\right)^{3} \leqq \sum_{k=1}^{\nu(n-1)}\left(r_{y, n-1}^{(k)}\right)^{3}
$$


From (14) again, we have for $n-1 \geqq N_{n}$,

$$
\left.\left.\sum_{k=1}^{\nu(n-1)}\left(r_{y, n-1}^{(k)}\right)^{3} \leqq \frac{1}{\left\{\Psi _ { 3 } ^ { - 1 } \left(\mu l_{3}^{1 / K}, n-1\right.\right.}\right)\right\}^{3} \sum_{k=1}^{\nu(n-1)}\left(\rho_{y, n-1}^{(k)}\right)^{3} .
$$

Bringing these into the right hand side of (16), we have for $n-1 \geqq N_{D}$,

$$
\sum_{j=1}^{\nu(n)}\left(\boldsymbol{r}_{y, n}^{(j)}\right)^{\alpha} \leqq \frac{\nu(n)^{1-\alpha / 3}}{\left\{\Psi_{3}^{-1}\left(\mu_{3, n}^{1 / K}\right)\right\}^{\alpha}\left\{\Psi_{3}^{-1}\left(\mu_{3, n-1}^{1 / K}\right)\right\}^{\alpha}}\left\{\sum_{k=1}^{\nu(n-1)}\left(\rho_{y, n-1}^{(k)}\right)^{3}\right\}^{\alpha / 3}
$$

This process can be continued up to $R_{y, N D}^{3(m)}$, and finally we obtain

$$
\sum_{j=1}^{\nu(n)}\left(r_{y, n}^{(j)}\right)^{\alpha} \leqq-\frac{\nu(n)^{1-\alpha / 3}}{\prod_{l=N D}^{n}\left\{\Psi_{3}^{-1}\left(\mu_{3, l}^{1 / K}\right)\right\}^{\alpha}}\left\{\sum_{m=1}^{\nu(N D)}\left(\rho_{y, N_{D}}^{(m)}\right)^{3}\right\}^{\alpha / 3}
$$

Starting from (15) and proceeding almost similarly as stated above, we have also

$$
\sum_{j=1}^{\nu(n)}\left(\gamma_{x, n}^{(j)}\right)^{\alpha} \leqq \frac{\nu(n)^{1-\alpha / 3}}{\prod_{l=1}^{n}\left\{\Psi_{3}^{-1}\left(\mu_{3, l}\right)\right\}^{\alpha}}\left\{\sum_{m=1}^{\nu(1)}\left(\rho_{x, 1}^{(m)}\right)^{3}\right\}^{\alpha / 3}
$$

Since $\Psi_{3}^{-1}(\mu) \geqq \Psi_{3}^{-1}\left(\mu^{1 / K}\right)$ for $\mu>1$ and

$$
\limsup _{n \rightarrow \infty} \prod_{l=1}^{n}\left\{\Psi_{3}^{-1}\left(\mu_{3, l}^{1 / K}\right)\right\}^{\alpha} / \nu(n)^{1-\alpha / 3}=+\infty
$$

from our assumption, it holds

$$
\liminf _{n \rightarrow \infty} \sum_{j=1}^{\nu(n)}\left(\boldsymbol{r}_{y, n}^{(j)}\right)^{\alpha}=0=\liminf _{n \rightarrow \infty} \sum_{j=1}^{\nu(n)}\left(\boldsymbol{r}_{x, n}^{(j)}\right)^{\alpha},
$$

which proves our assertion.

Corollary 8. Let $E_{x}$ and $y=y(x)$ be the same ones as in Theorem 7. If there exist a positive number $\delta$ and a system $\left\{R_{x, n}^{3\left(j_{1}\right)}(j=1,2, \ldots, \nu(n)<\infty ; n=1\right.$, $2, \ldots)$ which satisfy

$$
\liminf _{n \rightarrow \infty} \mu_{3, n}>\left\{\Psi_{3}(1+\delta)\right\}^{K} \text { and } \liminf _{n \rightarrow \infty} \frac{\nu(n)^{1-\alpha / 3}}{(1+\delta)^{n \alpha}}=0
$$

for some $\alpha$ such that $0<\alpha \leqq 3$, then the same conclusion as in Theorem 7 follow's.

15. Finally, we consider the particular case when for $E_{x}$ and $y=y(x)$ in Theorem $7, y=y(x)$ induces the correspondence between the plane Jordan domains on which $E_{x}$ and $y\left(E_{x}\right)$ lie respectively. 
THEOREM 8. Suppose that $y=y(x)$ is any K-quasiconformal mapping of a Jordan domain $D$ which transforms a plane Jordan domain $G$ included in $D$ into another plane Jordan domain $H$ and that $E_{x}$ is a compact set on $G$ such that the complement $\mathscr{C} E_{x}$ of $E_{x}$ in the extended plane on which $G$ lies a (connected) domain. If there exists a system $\left\{R_{n}^{2(j)}\right\}(j=1,2, \ldots, \nu(n)<\infty ; n=$ $1,2, \ldots)$ of (plane) circular rings inducing an exhaustion of the complement $\mathscr{C} E_{x}$ which satisfies

$$
\limsup _{n \rightarrow \infty}\left\{\alpha \sum_{l=1}^{n} \log \Psi_{3}^{-1}\left(\mu_{2, l}^{1 / K}\right)-\left(1-\frac{\alpha}{2}\right) \log \nu(n)\right\}=+\infty
$$

for some $\alpha$ such that $0<\alpha \leqq 2$, then $E_{x}$ and its image set $E_{y}$ by $y=y(x)$ are of $\alpha$-dimensional measure zero, where $\log \mu_{2, n}$ means $\min _{1 \leqq j \leqq v(u)} \bmod R_{x, n}^{2(j)}=\min _{1 \leqq j \leqq v(n)}$ $\log \mu_{x, n}^{2(j)}$.

Proof. First, construct the system $\left\{R_{x, n}^{3(j)}\right\}$ of spherical rings corresponding to the given system $\left\{R_{x, n}^{2(j)}\right\}$ of circular rings, and let $N_{D}$ be a number such that the sub-system of $\left\{R_{x, n}^{3(j)}\right\}$ for $n \geqq N_{D}$ is contained in $D$. Denote by $R_{y, n}^{2(j)}$ the intersection of the image $R_{y, n}^{3(j)}$ of $R_{x, n}^{3(j)}$ for $n \geqq N_{D}$ with the plane $H$. Then $\left\{R_{y, n}^{2(j)}\right\}\left(j=1,2, \ldots, \nu(n)<\infty ; n=N_{D}, N_{D}+1, \ldots\right)$ is evidently a system of rings inducing an exhaustion of the complement $\mathscr{C} E_{y}$ of $E_{y}$ in the extended plane on which $H$ lies.

Next put $\bmod R_{y, n}^{3(j)}=\log \mu_{y, n}^{3(j)}$ for $n \geqq N_{D}$, and take a point $y_{0, n}^{(j)}$ in the bounded complement $C_{0, n}^{2(j)}$ of $R_{y, n}^{2(j)}$, and put for $n \geqq N_{n}$,

$$
r_{y, n}^{(j)}=\max _{y \in B_{0, n}^{2(j)}}\left|y-y_{0, n}^{(j)}\right|, \rho_{y, n}^{(j)}=\min _{y \in B_{1, n}^{2(j)}}\left|y-y_{0, n}^{(j)}\right|,
$$

where $B_{0, n}^{2(j)}$ is the inner boundary of $R_{y, n}^{2(j)}$ and $B_{1, n}^{2(j)}$ the outer one, and define $r_{x, n}^{(j)}, \rho_{x, n}^{(j)}$ for $R_{x, n}^{2(j)}$ similarly to $r_{y, n}^{(j)}, \rho_{y, n}^{(j)}$, respectively. Then, the preceding relations (14) for $n \geqq N_{D}$ and (15) hold also in this case.

Applying Hölder's inequality to (14) and (15), and proceeding almost similarly to the previous argument, we have finally for $0<\alpha \leqq 2$,

and

$$
\sum_{j=1}^{\nu(n)}\left(\boldsymbol{r}_{y, n}^{(j)}\right)^{\alpha} \leqq \frac{\nu(n)^{1-\alpha / 2}}{\prod_{l=N_{D}}^{n}\left\{\Psi_{3}^{-1}\left(\mu_{3, l}^{1 / K}\right)\right\}^{\alpha}}\left\{\sum_{m=1}^{\nu(N D)}\left(\rho_{y, N D}^{(m)}\right)^{2}\right\}^{\alpha / 2}
$$

$$
\sum_{j=1}^{\nu(n)}\left(\gamma_{x, n}^{(j)}\right)^{\alpha} \leqq \frac{\nu(n)^{1-\alpha / 2}}{\prod_{l=1}^{n}\left\{\Psi_{3}^{-1}\left(\mu_{3, l}\right)\right\}^{\alpha}}\left\{\sum_{m=1}^{\nu(1)}\left(\rho_{x, 1}^{(m)}\right)^{2}\right\}^{\alpha / 2} .
$$


Since $\mu_{3, l}=\mu_{2, l}$ by Lemma 4 , we have $\Psi_{3}^{-1}\left(\mu_{3, l}\right) \geqq \Psi_{3}^{-1}\left(\mu_{3, l}^{1 / K}\right)=\Psi_{3}^{-1}\left(\mu_{2, l l}^{1 / K}\right) . \quad$ Further, from our assumption it follows that

$$
\liminf _{n \rightarrow \infty} \nu(n)^{1-\alpha / 2} / \prod_{l=1}^{n}\left\{\Psi_{3}^{-1}\left(\mu_{2, l}^{1 / K}\right)\right\}^{\alpha}=0,
$$

which implies our assertion.

Corollary 9. Let $E_{x}$ and $y=y(x)$ be the same ones as in Theorem 8. If there exist a positive number $\delta$ and a system $\left\{R_{x, n}^{2(j)}\right\}(j=1,2, \ldots, \nu(n)<\infty$; $n=1,2, \ldots)$ of circular rings $R_{x, n}^{2(j)}$ which satisfy

$$
\liminf _{n \rightarrow \infty} \mu_{2, n}>\left\{\Psi_{3}(1+\delta)\right\}^{K} \text { and } \liminf _{n \rightarrow \infty} \frac{\nu(n)^{1-\alpha / 2}}{(1+\delta)^{n \alpha}}=0
$$

for some $\alpha$ such that $0<\alpha \leqq 2$, then the same conclusion as in Theorem 8 follows.

We gave in [6] an example of the set, of positive logarithmic capacity, to which a system satisfying the condition in Theorem 8 or Corollary 9 corresponds. By the way, an example of the set with such a property in Theorem 7 or Corollary 8 is also given as follows.

Let $E\left(p_{1}, p_{2}, \ldots\right)$ be the symmetric Cantor set generated from a closed segment with length $l_{1}$, and let $S_{n+1}^{(j)}\left(j=1,2, \ldots, 2^{n}\right)$ be closed segments, with equal length $l_{n+1}$, remaining after the $n$-th deletion process taken in its generation. Denote by $R_{n}^{2(j)}$ the concentric circular ring whose center is the middle point of $S_{n+1}^{(j)}$ and whose radii $\frac{l_{n}}{4}\left(1+1 / p_{n+1}\right)\left(1-1 / p_{n}\right)$ and $\frac{l_{n}}{4}\left(1+1 / p_{n}\right)$. We can see that $\bmod R_{n}^{2(j)} \geqq 1 / 2\left(1-1 / p_{n}\right)$.

Now, take the direct product set $E\left(p_{1}, p_{2}, \ldots\right) \times E\left(p_{1}, p_{2}, \ldots\right) \times E\left(p_{1}\right.$, $\left.p_{2}, \ldots\right)$ and make each spherical ring $R_{n}^{3(j k l)}$, corresponding to each circular ring $R_{n}^{2(j)}$, which has the center at the middle point of each cube $S_{n+1}^{(j)} \times S_{n+1}^{(k)} \times$ $S_{n+1}^{(l)}$. Then we have a system $\left\{R_{n}^{3(j k l)}\right\}\left(j, k, l=1,2, \ldots, 2^{n} ; n=1,2, \ldots\right)$ inducing an exhaustion of the complement of the above direct product set in the Moebius space. In particular, it is easily shown that this system for $p_{n}=$ $3\left\{\Psi_{3}\left(8^{1 / \alpha}\right)\right\}^{K} /\left[3\left\langle\Psi_{3}\left(8^{1 / \alpha}\right)\right\}^{K}-1\right]$ satisfies the condition in Theorem 7 or Corollary 8 and the direct product set is of positive logarithmic capacity.

\section{References}

[1] Fuglede, B.: Extremal length and functional completion, Acta Math., 98 (1957), 171219. 
[2] Gehring, F. W.: Symmetrization of rings in space, Trans. Amer. Math. Soc., 101 (1961), 499-516.

[3] Gehring, F. W.: Rings and quasiconformal mappings in space, Trans. Amer. Math. Soc., 103 (1962), 353-393.

[4] Gehring, F. W.: Extremal length definitions for the conformal capacity of rings in space, Mich. Math. J., 9 (1962), 137-150.

[5] Ikoma, K. and Shibata, K.: On distortions in certain quasiconformal mappings, Tóhoku Math. J., 13 (1961), 241-247.

[6] Ikoma, K.: A criterion for a set and its image under quasiconformal mapping to be of $\alpha(0<\alpha \leqq 2)$-dimensional measure zero, Nagoya Math. J., 22 (1963), 203-209.

[7] Mori, A.: On quasi-conformality and pseudo-analyticity, Trans. Amer. Math. Soc., 84 (1957), 56-77.

[8] Pólya, G. and Szegö, G.: Isoperimetric inequalities in mathematical physics, Ann. of Math. Studies, No. 27, Princeton 1951.

[9] Šabat, B. V.: On the theory of quasiconformal mappings in space, Soviet Math. Dokl., 1 (1960), 730-733.

[10] Ugaheri T.: On the general capacities and potentials, Bull. Tokyo Inst Tech., 4 (1953), 149-179.

[11] Väisälä, J.: On quasiconformal mappings in space, Ann. Acad. Sci. Fenn., A. I. 298 (1961), 1-36.

[12] Väisälä, J.: On quasiconformal mappings of a ball, Ann. Acad. Sci. Fenn., A. I. 304 (1961), 1-7.

Department of Mathematics

Yamagata University 